\title{
Human Umbilical Cord Mesenchymal Stem Cells Therapy in Cyclophosphamide-Induced Premature Ovarian Failure Rat Model
}

\author{
Dan Song, ${ }^{1}$ Yun Zhong, ${ }^{1}$ Chunfeng Qian, ${ }^{1}$ Qinyan Zou, ${ }^{1}$ Jian Ou, ${ }^{1}$ Yichao Shi, ${ }^{1}$ \\ Liang Gao, ${ }_{1}^{1}$ Gaigai Wang, ${ }^{1}$ Zhenxing Liu, ${ }^{1}$ Haibo Li, ${ }^{1}$ Hailei Ding, ${ }^{2}$ Huihua Wu, \\ Fuxin Wang, ${ }^{1}$ Jing Wang, ${ }^{3}$ and Hong $\mathrm{Li}^{1}$ \\ ${ }^{1}$ Center for Reproduction and Genetics, Suzhou Municipal Hospital, Nanjing Medical University Affiliated Suzhou Hospital, \\ Suzhou, Jiangsu 215002, China \\ ${ }^{2}$ Jiangsu Beike Biotechnology Co., Ltd., Jiangsu 215002, China \\ ${ }^{3}$ Experimental Animal Center, Soochow University, Suzhou, Jiangsu 215006, China
}

Correspondence should be addressed to Hong Li; hongliszivf@163.com

Received 17 December 2015; Revised 27 January 2016; Accepted 2 February 2016

Academic Editor: Elena Jones

Copyright (C) 2016 Dan Song et al. This is an open access article distributed under the Creative Commons Attribution License, which permits unrestricted use, distribution, and reproduction in any medium, provided the original work is properly cited.

\begin{abstract}
Premature ovarian failure (POF) is one of the most common causes of infertility in women. In our present study, we established cyclophosphamide- (CTX-) induced POF rat model and elucidated its effect on ovarian function. We detected the serum estrogen, follicle stimulating hormone, and anti-Müllerian hormone of mice models by ELISA and evaluated their folliculogenesis by histopathology examination. Our study revealed that CTX administration could severely disturb hormone secretion and influence folliculogenesis in rat. This study also detected ovarian cells apoptosis by deoxy-UTP-digoxigenin nick end labeling (TUNEL) and demonstrated marked ovarian cells apoptosis in rat models following CTX administration. In order to explore the potential of human umbilical cord mesenchymal stem cells (UCMSCs) in POF treatment, the above indexes were used to evaluate ovarian function. We found that human UCMSCs transplantation recovered disturbed hormone secretion and folliculogenesis in POF rat, in addition to reduced ovarian cell apoptosis. We also tracked transplanted UCMSCs in ovaries by fluorescence in situ hybridization (FISH). The results manifested that the transplanted human UCMSCs could reside in ovarian tissues and could survive for a comparatively long time without obvious proliferation. Our present study provides new insights into the great clinical potential of human UCMSCs in POF treatment.
\end{abstract}

\section{Introduction}

Premature ovarian failure (POF) is a heterogeneous disorder, which is defined as the cessation of ovarian function, along with elevated follicle stimulating hormone (FSH) and decreased estrogen (E2) levels in women below 40 years [1]. Ovarian atrophy, reduced follicle stores, menstrual irregularities, and ovarian dysfunction are the most important characteristics of POF. It has become an important cause of infertility. The etiology of POF is complex, which is often caused by genetic defects, autoimmunity, and toxics [2-4]. Nowadays, with the increasing cancer incidence among young women, long term exposure to gonadotoxicity chemotherapy is becoming a major cause of POF.
Cyclophosphamide (CTX), an alkylating agent, has been found to have a deleterious effect on female reproductive organs, especially on ovary [5]. The reversibility of the damage depends on patient's age, exposure degree, and ovarian reserve. The attempts to preserve or restore patients' fertility before or after oncologic therapy have always been a concern.

Gonadotropin and steroid hormone are the key regulators of folliculogenesis in all mammalian species [6]. Disordered endocrine system regulation affects the process of follicular development, follicular storage, and menopausal onset and leads to ovarian pathological conditions, such as POF [7]. Studies have shown that the reduced number of primordial and early antral follicles could reduce serum anti-Müllerian 
hormone $(\mathrm{AMH})$ level, which reflects follicular storage and is a reliable predictor of POF other than FSH and E2 $[8,9]$.

Stem cell transplantation has become a promising tool in rescuing damaged ovarian function. Its therapeutic potential has opened up a new way for preserving or recovering the damaged fertility of women receiving chemotherapy [10]. The application of stem cells transplantation is often associated with controversies, such as safety, source, and their poor survival time in vivo. Umbilical cord is a rich source of mesenchymal stem cells [11] and the umbilical cord mesenchymal stem cells (UCMSCs) are multipotent, nonhaematopoietic progenitor cells, which can differentiate into multiple cell lineages [12]. The phenotype and characteristic of UCMSCs, as identified by many studies, have many advantages over other MSCs [13-15]. Although its exact protective role in the injured tissues is still obscure, more and more studies have implied that it could inhibit stromal cell apoptosis by secreting growth factors [16-18].

In our current study, we established chemotherapy induced POF rat models, which represent a reliable and physiological model for studying the role of CTX in murine ovarian function. We investigated the potential therapeutic efficiency of human male-derived UCMSCs in rescuing the POF rats. Our study helps point out the potential of UCMSCs in POF treatment and the involved mechanism. We have exhibited the study design in a table (supplementary table 1 in Supplementary Material available online at http://dx.doi.org/10.1155/2016/2517514).

\section{Materials and Methods}

2.1. Experimental Animals. Eight-week-old female specific pathogen-free- (SPF-) grade Wistar rats, weighing 180-200 g, were used in our study. The rats were purchased from Soochow University (Suzhou, China). All the animals were housed in animal facility of Soochow University and were fed a standard pellet diet with free access to water. All the experimental procedures were conducted in accordance with the Institutional Animal Care and Use Committee at Soochow University.

2.2. Animal Model Establishment. To establish chemotherapy induced POF rat model, rats were randomly divided into two groups: control group and POF group. The POF group rats $(n=20)$ were intraperitoneally injected with $200 \mathrm{mg} / \mathrm{kg}$ of CTX (Endoxan, Shionogi \& Co., Osaka, Japan) on the first day and then $8 \mathrm{mg} / \mathrm{kg} /$ day for the 15 consecutive days. For the control group, we set the blank control group $(n=10)$ without any treatment and negative control group $(n=10)$, which was injected with $0.9 \%$ saline instead of CTX.

\subsection{Human UCMSCs Isolation and Phenotype Determination.} UCMSCs were obtained from Beike Biotech Co., Ltd., Jiangsu province. The preparation was as follows: Full-term umbilical cord (UC) was obtained after UC blood removal from newborn boy of normal deliveries after obtaining the informed consent. The isolated UC was manually dissected into 1$2 \mathrm{~mm}^{3}$ sections and incubated with $0.075 \%$ collagenase type II for $30 \mathrm{~min}$ and then $0.125 \%$ trypsin for $30 \mathrm{~min}$ with gentle agitation at $37^{\circ} \mathrm{C}$. UCs were plated at a density of $1 \times 10^{6} / \mathrm{cm}^{2}$ in cell culture dish containing L-DMEM, supplemented with $5 \%$ fetal bovine serum and $1 \%$ penicillin/streptomycin at $37^{\circ} \mathrm{C}$ in a humidified atmosphere of $5 \% \mathrm{CO}_{2}$. After three days, the nonadherent cells were removed. The first colonies of UCMSCs were obtained after changing the medium several times. Passage 2 cells were harvested with $0.25 \%$ trypsinEDTA and were disaggregated into single cell for further analysis and cells transplantation.

Flow cytometry was used to characterize the human UCMSCs. The following monoclonal antibodies (mAbs) were used: phycoerythrin (PE) conjugated anti-human mAbs against CD29/CD73/CD34 (CD29/CD73: BD Pharmingen, USA; CD34: BD eBioscience, USA); fluorescein isothiocyanate (FITC) conjugated anti-human mAbs against CD90/CD45/CD14 (CD90: BioLegend, USA; CD45/CD14: $\mathrm{BD}$ eBioscience, USA); allophycocyanin (APC) conjugated anti-human mAbs against CD105/CD79a (CD105: BD eBioscience, USA; CD79a: BD Pharmingen, USA); and peridininchlorophyll protein (perCP) complex conjugated anti-human mAbs against HLA-DR (BD eBioscience, USA).

The harvested UCMSCs were washed twice with FBScontaining PBS and were separately incubated with the above $\mathrm{mAbs}$ or mouse IgG isotype control for $30 \mathrm{~min}$ at $4^{\circ} \mathrm{C}$. Extra mAbs were removed by washing twice with PBS. The cells were resuspended in $0.5 \mathrm{~mL}$ PBS to obtain a final concentration of $2 \times 10^{5}$ cells and were analyzed by FACSCalibur Flow Cytometer (BD FACSCalibur).

2.4. Stem Cell Transplantation. In order to perform the UCMSCs transplantation, we established the POF rat model in the very same way as previous one. Two weeks after CTX administration, rats were divided into four groups. Group I included rats without any treatment; group II included POF rats model without UCMSCs transplantation; group III included POF rats model injected with male UCMSCs by tail intravenously; and group IV included POF rats model transplanted with UCMSCs in situ, with eight rats for each group. Prior to the UCMSCs transplantation, the rats were anesthetized by intraperitoneal injection of ketamine (45 mg/kg body weight). UCMSCs (passage 2 cells) were stored at $4^{\circ} \mathrm{C}$ in $\mathrm{PBS}$ for less than $30 \mathrm{~min}$ before transplantation. For tail intravenous trans group, POF rats were transplanted with UCMSCs $(100 \mu \mathrm{L}$, at a concentration of $1 \times$ $10^{6} / \mathrm{mL}$ ) intravenously with a microinjector. For in situ trans group, POF rats were transplanted directly through each of the bilateral ovaries with UCMSCs $(25 \mu \mathrm{L}$, at a concentration of $2 \times 10^{6} / \mathrm{mL}$ ) with a microinjector $[19,20]$.

2.5. Hormone Assay. To detect the levels of serum E2, FSH, and $\mathrm{AMH}$, blood samples were obtained from rats models by retroorbital puncture under anesthesia. Samples were incubated at room temperature for $1 \mathrm{~h}$, and supernatant was collected following centrifugation at $4000 \mathrm{r} / \mathrm{min}$ for $10 \mathrm{~min}$. The concentration of the hormone was determined by ELISA kits (Yison Bio, Shanghai, China).

According to the kit instructions, rat E2, FSH, and $\mathrm{AMH}$ standards were diluted by standard diluent at the final 
concentration of $0,5.0,10,20,40$, and $80 \mathrm{ng} / \mathrm{L}, 0,1.5,3$, 6,12 , and $24 \mathrm{IU} / \mathrm{L}$, and $12.5,25,50,100$, and $200 \mathrm{pg} / \mathrm{mL}$, respectively. Fifty microlitres of standard samples was added to the precoated microtest wells and $10 \mu \mathrm{L}$ plasma was added to $40 \mu \mathrm{L}$ sample diluent. The whole setting was incubated for $30 \mathrm{~min}$ at $37^{\circ} \mathrm{C}$. After washing for five times, $50 \mu \mathrm{L}$ HRP-conjugated reagent was added and the setting was incubated for another $30 \mathrm{~min}$ at $37^{\circ} \mathrm{C}$. After washing five times, tetramethyl benzidine (TMB) substrate was added to the well, and OD values of the samples were determined by TECAN infinite F80 at a wavelength of $450 \mathrm{~nm}$. According to the standard curve, the concentration of the hormones was determined.

\subsection{Ovarian Follicle Counting and Morphological Analysis} Using Hematoxylin and Eosin Staining. Rats were killed and ovaries were collected at different time intervals following the CTX administration. Ovary tissues were fixed in $10 \%$ paraformaldehyde for at least 24 hours. The ovaries were dehydrated and embedded in paraffin and were serially sectioned at $5 \mu \mathrm{m}$ depth; the sections were stained with hematoxylin and eosin (H\&E) for histopathology. The ovarian histological examination was performed using light microscopy (OLYMPUS, Japan). The follicles were detected and classified as primordial, primary, secondary, and early antral follicles, according to the previous description and definition [21]. Follicles containing an oocyte, with a clearly visible nucleus, were counted only.

2.7. Apoptosis Assay. In order to detect the cell apoptosis in rat ovaries, In Situ Cell Death Detection Kit (KeyGEN BioTech) was used to detect the fragmented DNA histochemically by TUNEL. Fluorescein-labeled nucleotides were incorporated in situ onto $3^{\prime}$ ends of DNA strand breaks of the apoptotic cells. According to the instruction, the paraffin sections were dewaxed at $60^{\circ} \mathrm{C}$ for $60 \mathrm{~min}$ and rehydrated with ethanol $(100 \%, 90 \%, 80 \%$, and $75 \%)$ in step by step manner. The slides were washed thrice with PBS for $5 \mathrm{~min}$ and incubated with proteinase $\mathrm{K}$ at $37^{\circ} \mathrm{C}$ for $30 \mathrm{~min}$. Fiftymicrolitre TdT enzyme reaction mixture was added to the samples, and the whole setting was incubated for $30 \mathrm{~min}$ at $37^{\circ} \mathrm{C}$ in a humidified atmosphere in the dark and washed thrice in PBS for $5 \mathrm{~min}$. Streptavidin-fluorescein reagent was added to the sections and incubated at $37^{\circ} \mathrm{C}$ in a humidified atmosphere for $30 \mathrm{~min}$ in the dark. After washing thrice with PBS, the cell nucleus was dyed with DAPI for $10 \mathrm{~min}$. Apoptotic cells in the ovary were stained green. The sections were observed with fluorescence microscopy (ZEISS Imager A1, Germany).

2.8. Stem Cell-Tracking. To determine the location of the transplanted UCMSCs and their fate in ovarian tissues, FISH was performed. Rats were killed at six weeks and eight weeks after UCMSCs transplantation. Ovaries were collected and were fixed in $10 \%$ paraformaldehyde for at least 24 hours. The ovaries were sectioned into paraffin with a depth of $5 \mu \mathrm{m}$. PathVysion Probe Kit Vysis LSI SRY_Spectrum Orange/CEPX Spectrum Green (Abbott Laboratories, Abbott Park) was used to mark human UCMSCs in ovarian tissue.
According to the instruction, the paraffin sections were dewaxed at $60^{\circ} \mathrm{C}$ for 6 hours and were further dewaxed by treatment of dimethylbenzene three times for $10 \mathrm{~min}$ and rehydrated three times with ethanol $100 \%$ for $5 \mathrm{~min}$. The sections were denatured with Pretreatment Reagent Kit and were incubated in $80^{\circ} \mathrm{C}$ for $20 \mathrm{~min}$ and were washed with $\mathrm{ddH}_{2} \mathrm{O}$. The sections were incubated in pepsin at $37^{\circ} \mathrm{C}$ for $15 \mathrm{~min}$ and washed with $\mathrm{ddH}_{2} \mathrm{O}$. Sections were dehydrated in ethanol $(70 \%, 85 \%$, and $100 \%)$ separately for $1 \mathrm{~min}$. Probe preparation: the sections were denatured at $73^{\circ} \mathrm{C}$ for $10 \mathrm{~min}$ and were incubated at $37^{\circ} \mathrm{C}$ for $7 \mathrm{~min}$. Sections were hybridized with probe at $37^{\circ} \mathrm{C}$ for $16 \mathrm{~h}$. Following this, the sections were washed in buffer ( $2 x$ SSC/0.3\% NP-40) for 3 min. Counterstaining was carried out with 4,6-diamidino2-phenylindole (DAPI) for $10 \mathrm{~min}$ in the dark. All the sections were visualized using fluorescence microscopy (OLYMPUS, BX53). The location of the UCMSCs was determined using orange (SRY/Y chromosome) and green (CEP/X chromosome) signals.

2.9. Statistical Analyses. The follicle numbers were expressed as mean \pm standard deviation (SD) and were analyzed by Student's $t$-test and one-way ANOVA. Plasma hormone levels at different time point in each group were analyzed by repeated measures ANOVA, and multiple comparisons of different groups were also conducted. $p$ value of $<0.05$ was considered to be statistically significant for all of the involved groups, and all analyses were conducted with software of SPSS 16.0 .

\section{Results}

3.1. Isolation and Characterization of Human UCMSCs. The surface molecular expression of the enriched human UCMSCs was detected using flow cytometry following 2 passages. The results indicated a high expression of CD90/CD29/CD73/CD105 (>95\%) for the isolated human UCMSCs (Figures 1(a), 1(b), 1(c), and 1(e)). FACS analysis also reported very low expression levels of CD45/CD34/CD79a/CD14/HLA-DR (<2\%) (Figures 1(d), 1(f), 1(g), 1(h), and 1(i)).

3.2. Effect of CTX on Ovarian Morphology. All the animals were weighed before and after the CTX treatment and no significant difference was observed between them. However, although not quantitatively measured, the reduction in the size of reproductive organs (ovaries and uterus) was observed after the CTX treatment (Figure 2). Comparing with the control group, the ovaries and uterus of POF rats were atrophied after two weeks of CTX administration.

3.3. Chemotherapy Induced Serum Hormone Level Changes of POF Model. Blood was obtained from the same four rats of each group after CTX administration for one week, two weeks, and three weeks, respectively. The serum levels of $\mathrm{FSH}, \mathrm{E} 2$, and $\mathrm{AMH}$ were all significantly different among these three groups (blank control group, saline treatment group, and CTX treatment group) ( $p=0.001, p=0.032$, and $p<0.0001$, resp.). The serum FSH level in CTX 

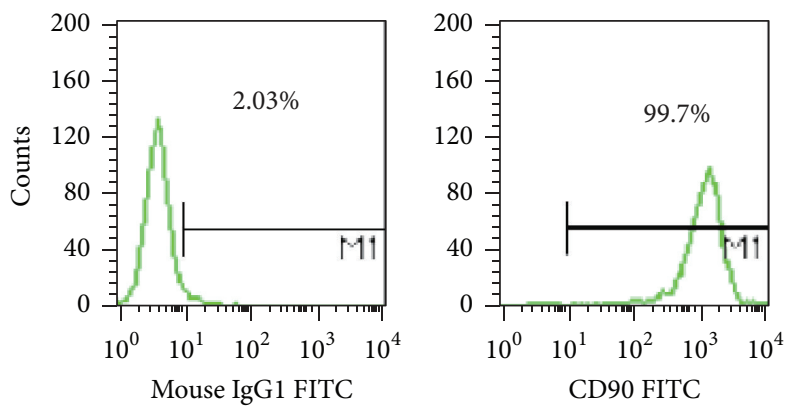

(a)

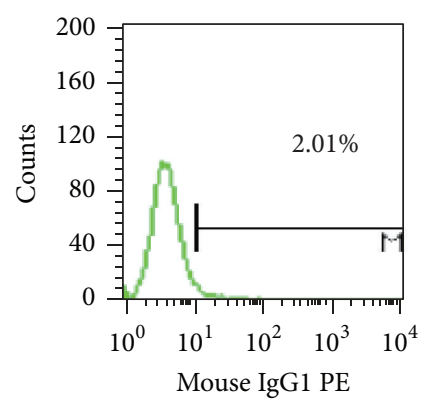

(c)
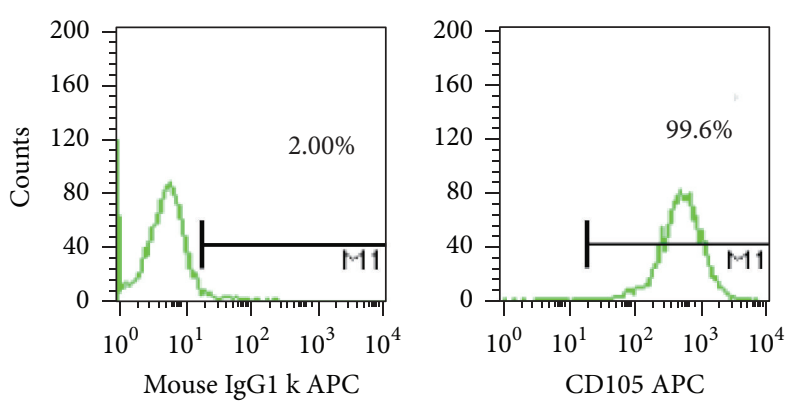

(e)
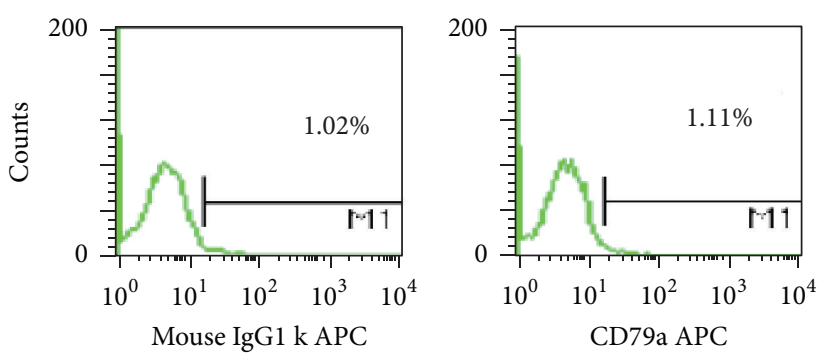

(g)

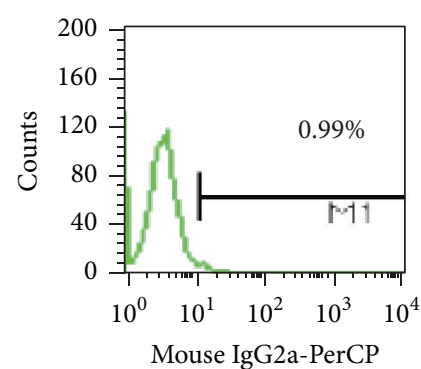

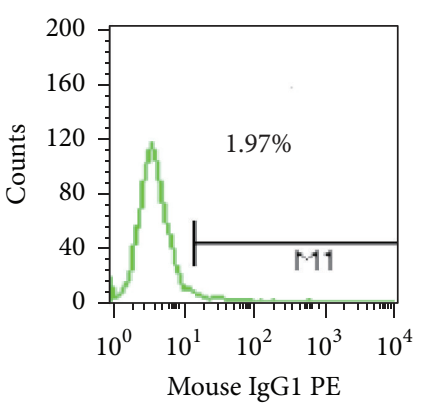

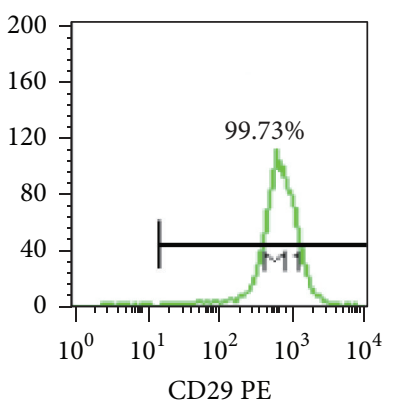

(b)
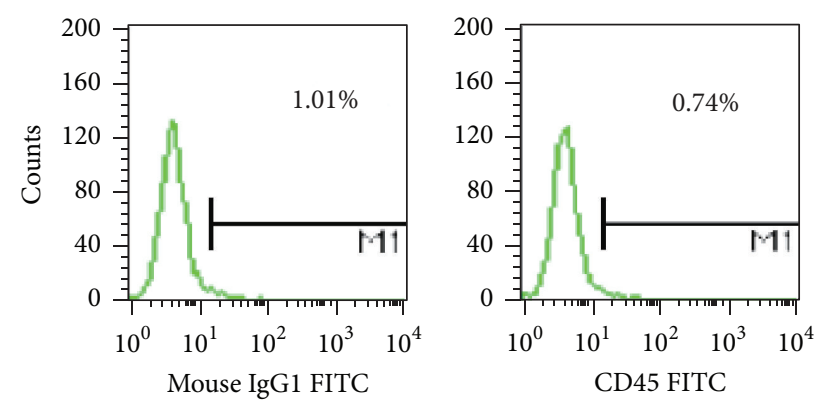

(d)
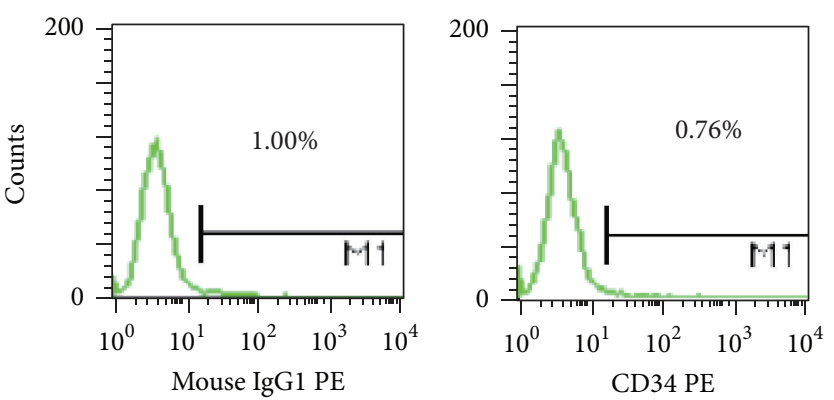

(f)
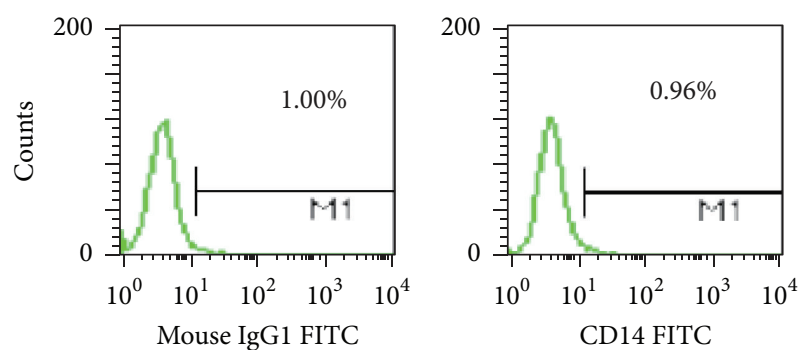

(h)

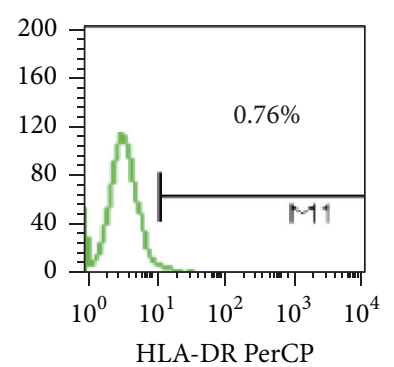

(i)

FIGURE 1: Flow cytometry analysis of human UCMSCs. Flow cytometry analysis of human UCMSCs showed their expression for CD90/CD29/ CD73/CD45/CD105/CD34/CD79a/CD14/HLA-DR. 
TABLE 1: Serum levels of FSH, AMH, and E2 determined by ELISA at different CTX posttreatment intervals in three groups (blank control group, saline treatment group, and CTX treatment group). Data were expressed as means \pm SD $(n=4)$.

\begin{tabular}{|c|c|c|c|c|}
\hline & 0 weeks & 1 week & 2 weeks & 3 weeks \\
\hline \multicolumn{5}{|l|}{ FSH (IU/L) } \\
\hline Blank control & $12.559 \pm 1.485$ & $12.294 \pm 1.096$ & $12.131 \pm 0.834$ & $13.294 \pm 0.334$ \\
\hline Saline control & $10.621 \pm 0.987$ & $12.303 \pm 1.292$ & $13.690 \pm 0.941$ & $12.780 \pm 1.239$ \\
\hline CTX treatment & $12.661 \pm 0.404$ & $14.401 \pm 0.946$ & $15.467 \pm 0.782$ & $18.164 \pm 1.660$ \\
\hline \multicolumn{5}{|l|}{$\mathrm{E} 2(\mathrm{ng} / \mathrm{L})$} \\
\hline Blank control & $52.146 \pm 12.187$ & $61.213 \pm 4.385$ & $53.824 \pm 4.080$ & $52.349 \pm 10.963$ \\
\hline Saline control & $52.532 \pm 3.097$ & $55.330 \pm 4.087$ & $52.601 \pm 9.040$ & $51.927 \pm 4.936$ \\
\hline CTX treatment & $49.284 \pm 5.980$ & $40.298 \pm 7.885$ & $38.095 \pm 4.475$ & $35.356 \pm 4.567$ \\
\hline \multicolumn{5}{|l|}{$\mathrm{AMH}(\mathrm{pg} / \mathrm{mL})$} \\
\hline Blank control & $166.288 \pm 11.144$ & $152.035 \pm 9.665$ & $174.385 \pm 14.816$ & $188.845 \pm 21.469$ \\
\hline Saline control & $176.817 \pm 15.977$ & $165.875 \pm 12.584$ & $161.025 \pm 13.914$ & $174.635 \pm 18.181$ \\
\hline CTX treatment & $180.490 \pm 13.310$ & $129.340 \pm 14.390$ & $153.680 \pm 17.830$ & $139.420 \pm 7.700$ \\
\hline
\end{tabular}

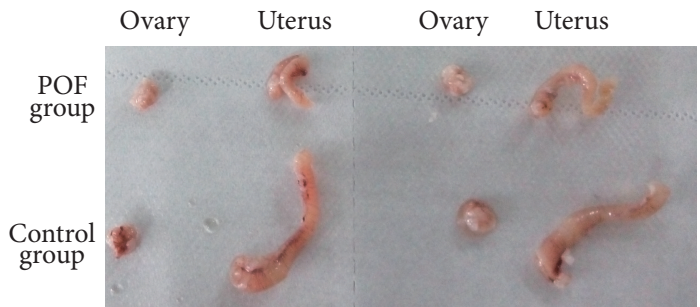

FIGURE 2: Photographs of ovaries removed from CTX treated groups and control group.

treatment group was significantly increased comparing with that in blank control group $(p=0.001)$ (Figure 3(a)). The serum E2 level in CTX treatment group was significantly decreased comparing with that in blank control group $(p<$ 0.0001) (Figure 3(b)). AMH level in CTX treatment group was significantly decreased comparing with that in blank control group $(p=0.019)$ (Figure 3(c)). The level of FSH and AMH at different observed times was significantly different ( $p<0.0001, p<0.0001$, resp.), while E2 level was not significantly different at different observed times $(p=0.227)$. Considering that injection process would be an interference factor during CTX administration, we set saline treatment group. According to our study, there was no significant difference in the hormones level between blank control group and saline treatment group $(p>0.05)$; thus the saline treatment group was not included in the following experiments. The data has been presented as Table 1 .

3.4. Effect of CTX on Follicle Development. Four weeks after CTX administration, three rats from each group were sacrificed for ovarian pathology analysis. In order to detect the effect of CTX administration on the total number of follicles in every stage, we randomly selected five slices of every ovary and classified and counted the follicles on the entire field of these five slices. We separately added the number of follicles in the same stage of these five slices together. Mean of the number of every stage's follicles for two ovaries from one rat was calculated and used for further analysis. We separately calculated the mean numbers of follicles in every stage for three rats per group and perform statistical comparisons.

Follicles of every stage were classified according to the following criteria: primordial follicles were identified as follicles with oocytes surrounded by less differentiated squamous granulosa cells (GCs); primary follicles were identified as those with oocytes surrounded by a single layer of cuboidal GCs; secondary follicles were identified as those with oocytes surrounded by two to four complete GC layers; and early antral follicles were identified as those with oocytes surrounded by five or more complete GC layers, with or without a visible antrum. Our results indicated that the number of secondary follicles was significantly decreased in the CTX treatment group ( $p=0.004$ ), while no significant difference was observed in the number of follicles in other stages between CTX group and control group (Figure 4). The data has been presented in Table 2 .

\subsection{UCMSCs Transplantation Improved Hormone Secretion in} POF Rats. Blood was obtained from each rat in all groups at two weeks, four weeks, and six weeks after UCMSCs transplantation. The effects of UCMSCs transplantation on the hormone secretion (FSH, E2, and $\mathrm{AMH}$ ) of rats were analyzed. Effect indicators were compared among CTX treatment group and UCMSCs transplantation groups for all of these time points. The serum FSH, E2, and AMH levels were all significantly different among these groups (blank control group, CTX control group, and UCMSCs trans groups) ( $p=$ $0.004, p=0.001$, and $p<0.0001$, resp.). Serum FSH of blank control group maintained a significantly low level ( $p=$ 0.001 ), with its E2 and AMH maintaining a significantly high level $(p<0.0001)$, comparing with that in CTX control group. The serum FSH level decreased significantly after UCMSCs transplantation both in tail trans group and in in situ trans group comparing with that in CTX control group $(p=0.001, p=0.009$, resp.) (Figure 5(a)). The serum E2 level has a significant recovery after UCMSCs transplantation for both trans groups comparing with that in CTX control group $(p<0.0001)$ (Figure 5(b)). Our study reported that UCMSCs 


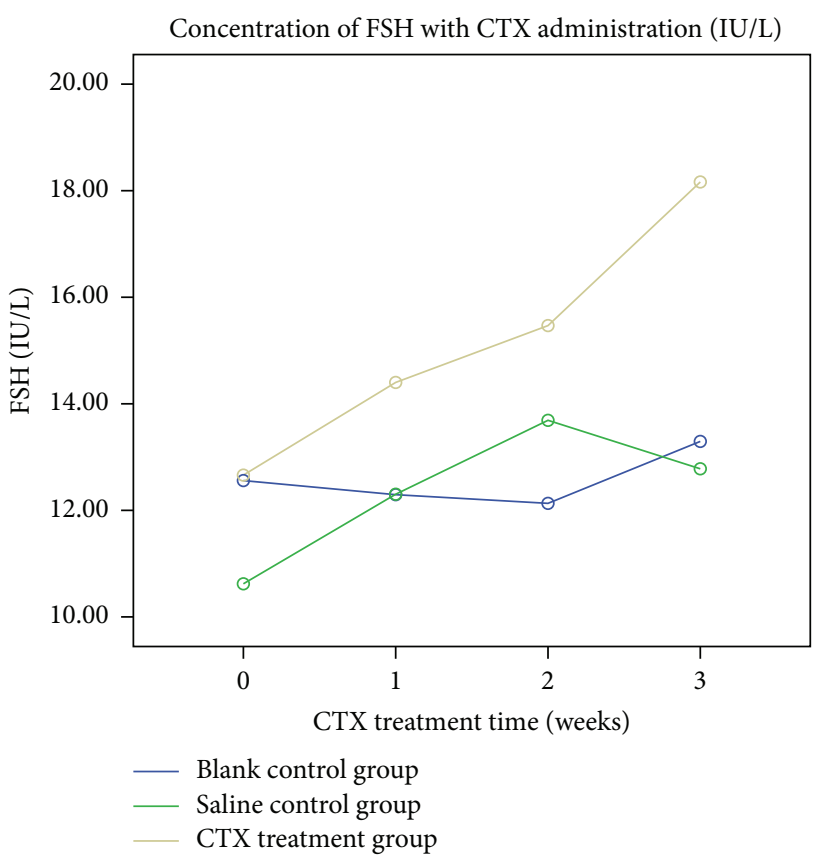

(a)

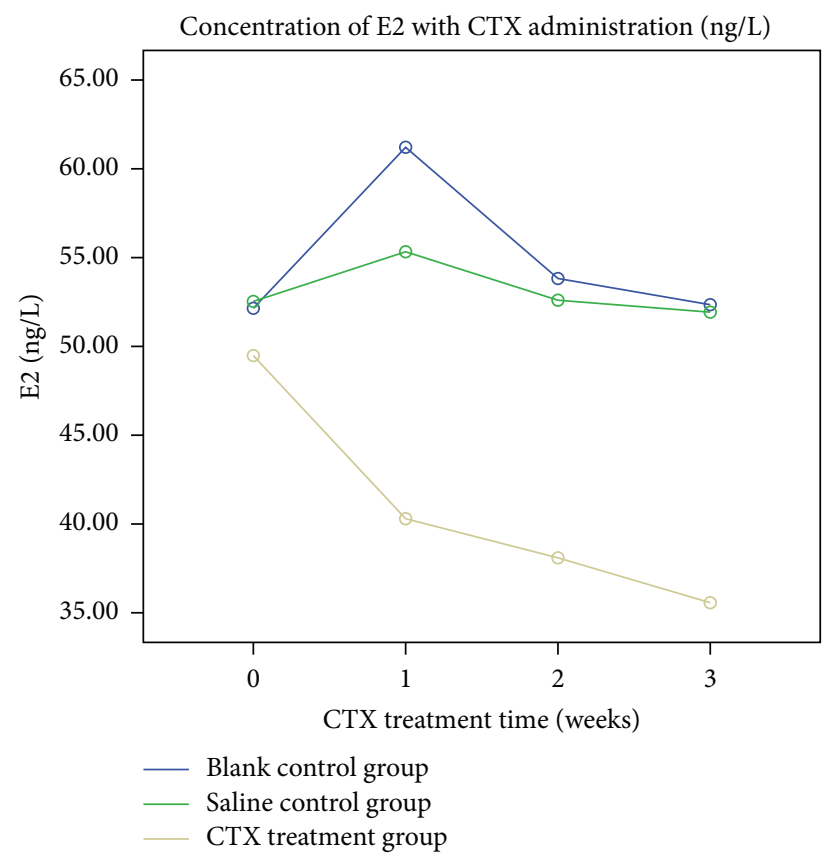

(b)

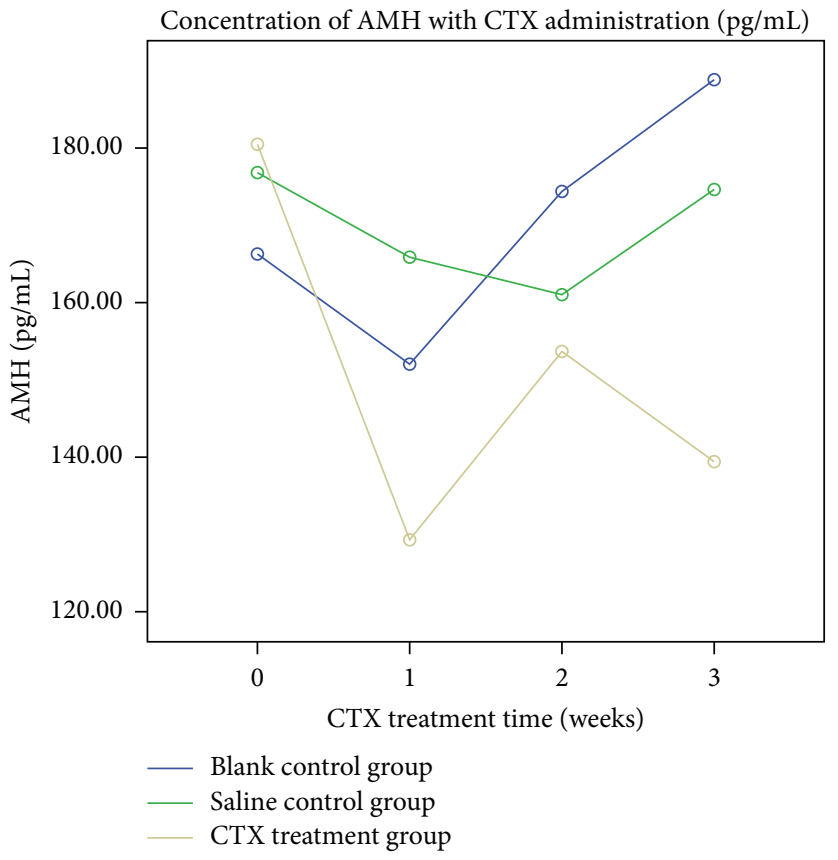

(c)

FIGURE 3: The trend for average level of FSH, AMH, and E2 in each group (blank control group, saline treatment group, and CTX treatment group) during three weeks after CTX treatment.

transplantation has a significant effect on AMH levels in POF rat models for tail trans group comparing with that in CTX control group ( $p=0.023$ ), but not for in situ trans group $(p>0.05)$ (Figure 5(c)). The level of E2 and AMH at different observed time point was significantly different $(p=0.002$, $p=0.002$, resp.), while FSH level was not significantly different at different observed time points $(p>0.05)$. The data has been presented in Table 3.
3.6. Effect of UCMSCs Transplantation on Follicle Development. Three rats of each group were killed at 6 weeks after UCMSCs transplantation. All the stages of follicles (primordial, primary, secondary, and early antral follicles) were analyzed (Figure 6). The same follicle counting method and statistical comparison method were adopted as those used in Effect of CTX on Follicle Development. Our results indicated a significant increase in secondary follicles in UCMSCs trans 
TABLE 2: Counts of different stages follicles were presented as means $\pm \mathrm{SD}(n=3)$ (primordial, primary, and early antral follicles of CTX treatment group versus blank control group: $p>0.05$, secondary follicles of CTX treatment group versus blank control group: $p=$ $0.004)$.

\begin{tabular}{lcccc}
\hline & Primordial & Primary & Secondary & Early antral \\
\hline Blank control & $40.7 \pm 9.2$ & $14.3 \pm 2.5$ & $8.3 \pm 1.5$ & $20.3 \pm 4.5$ \\
CTX treatment & $23.0 \pm 1.7$ & $15.0 \pm 6.2$ & $4.7 \pm 1.5$ & $28.3 \pm 3.8$ \\
\hline$p$ & $\mathrm{~ns}$ & $\mathrm{~ns}$ & 0.004 & $\mathrm{~ns}$ \\
\hline
\end{tabular}

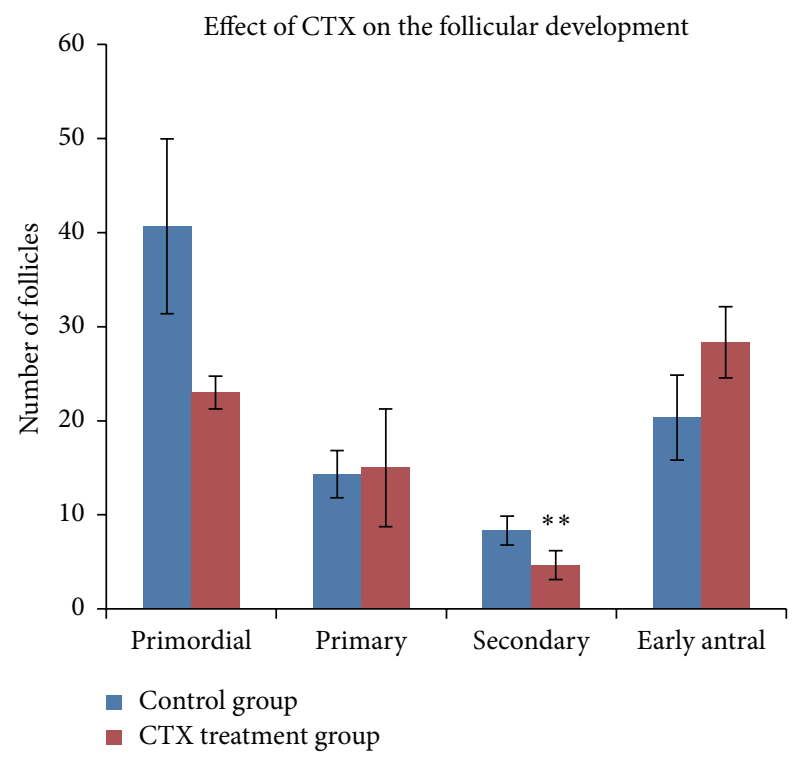

FIGURE 4: Effect of CTX on the follicles development. Ovarian H\&E staining pathological sections were from control group and CTX treatment group. Follicles were counted and classified. Data were means \pm SD of counts of different stages follicles $(n=3)$ (primordial, primary, and early antral follicles of POF group versus control group: $p>0.05$, secondary follicles of POF group versus control group: $p=0.004) .{ }^{* *} p<0.01$.

groups (both in tail intravenous group and in in situ group) as compared to POF group without UCMSCs transplantation (blank control group versus CTX control group, $p<0.0001$; tail trans group versus CTX control group, $p=0.001$; and in situ trans group versus CTX control group, $p=0.003$ ); however, no significant change was observed in the number of primordial follicles, primary follicles, and antral follicles among all the groups $(p>0.05)$. The data has been presented in Table 4.

3.7. UCMSCs Transplantation Reduced Ovarian Cells'Apoptosis in POF Rats. Cells apoptosis in ovarian tissues was detected by TUNEL. A number of apoptotic cells were observed in the ovarian tissues of CTX treatment group; ovaries from control group showed mostly healthy follicles with no sign of mounts of cells apoptosis, which was attributed to UCMSCs transplantation. According to our results, after two weeks of UCMSCs transplantation (both in situ trans group and tail intravenous trans group), the number of apoptosis cells (FITC-positive cells) in ovary sections was reduced as compared to that in nontrans group (POF group) (Figure 7).

3.8. Human UCMSCs Infiltrated into Rat Ovarian Tissue. FISH analysis showed the spatial location of transplanted human UCMSCs in ovary tissues. The analysis tracked the fate of the transplanted human UCMSCs at different time points in ovary. Double-labeled staining (red and green fluorescence) cells were defined as human UCMSCs (Figure 8). UCMSCs detected in the ovarian tissues were derived from UCMSCs transplanted groups at six and eight weeks after UCMSCs transplantation. The amount of UCMSCs in the rat ovarian tissue was basically constant, without obvious proliferation during this period, both in tail intravenous trans group and in situ trans group. The UCMSCs, however, were not detected in blank control group and POF control group (nontrans group).

\section{Discussion}

The potential of chemotherapy in inducing POF has attracted more concern recently $[22,23]$. Since chemotherapy agents could trigger cells into a programmed cell death pathway, continual renewal of ovarian cells makes them more vulnerable to chemotherapy agents. In our study, we observed that CTX administration significantly reduced the ovarian size of POF rats. Ovarian GCs are the most important stroma cells in ovary, which surround oocyte and play a key role in folliculogenesis [24]. Based on its potential of secreting growth factors and providing hormone supplementation, GCs are critical for oocyte growth and survival [25]. The development of follicles always comes along with active GC proliferation and apoptosis and in deciding the fate of follicles in adult human ovaries. Overapoptosis of GCs induced by chemotherapy is the main cause of follicular over-atresia and POF [26]. Our results revealed that chemotherapy severely induced GCs' apoptosis in POF rat models. Further, ovarian pathological analysis presented that CTX administration significantly decreased the number of secondary follicles, while having not much influence on follicles in other stages. Such a phenomenon may be due to the blooming GCs proliferation in this stage. Studies have shown that gonadotropin-releasing hormone analog (GnRHa) may protect the ovary from the effect of chemotherapy by inhibiting its proliferation and follicles growth [27].

Stem cells transplantation is an ideal potential treatment for repairing injured tissue and is also a powerful tool in restoring fertility and pregnancy [28]. Various types of stem cell have been investigated in POF treatment, such as adipose-derived stem cells (ADSCs) [29], human amniotic fluid-derived stem cells (hAFCs) [30], and skin-derived mesenchymal stem cells (SMSCs) [31]. Although an ideal and promising treatment for POF, stem cell transplantation also has some debate going on about its exact mechanism. Some reports supported the fact that transplanted stem cells could be induced to differentiate into ovarian tissue-like cells [32], while others have supported stem cells could help improve the damaged ovarian niches. 


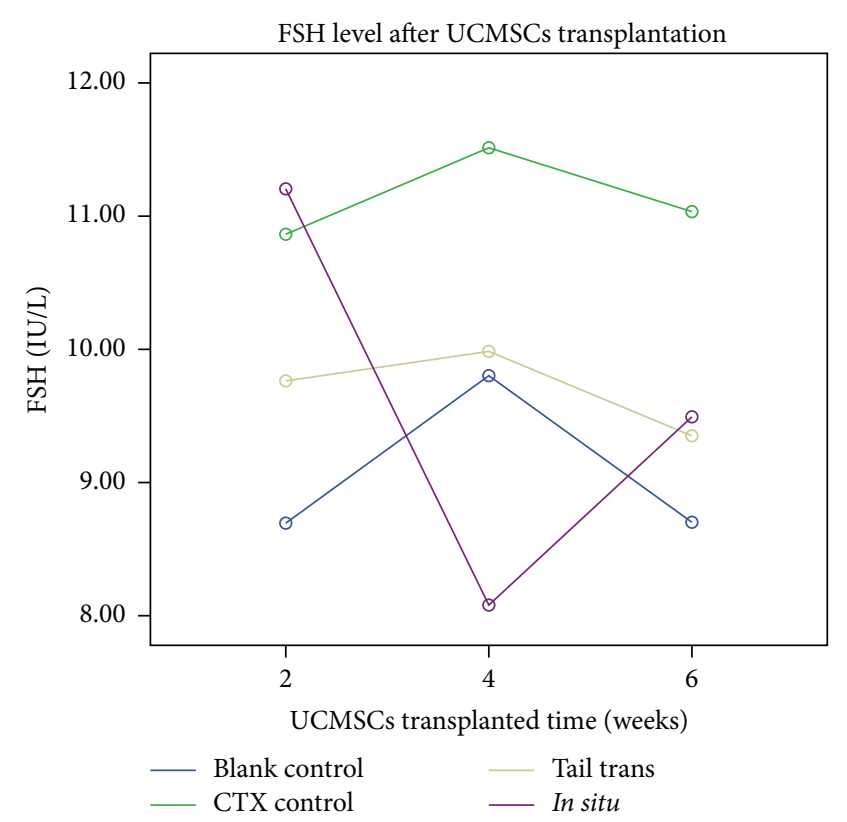

(a)

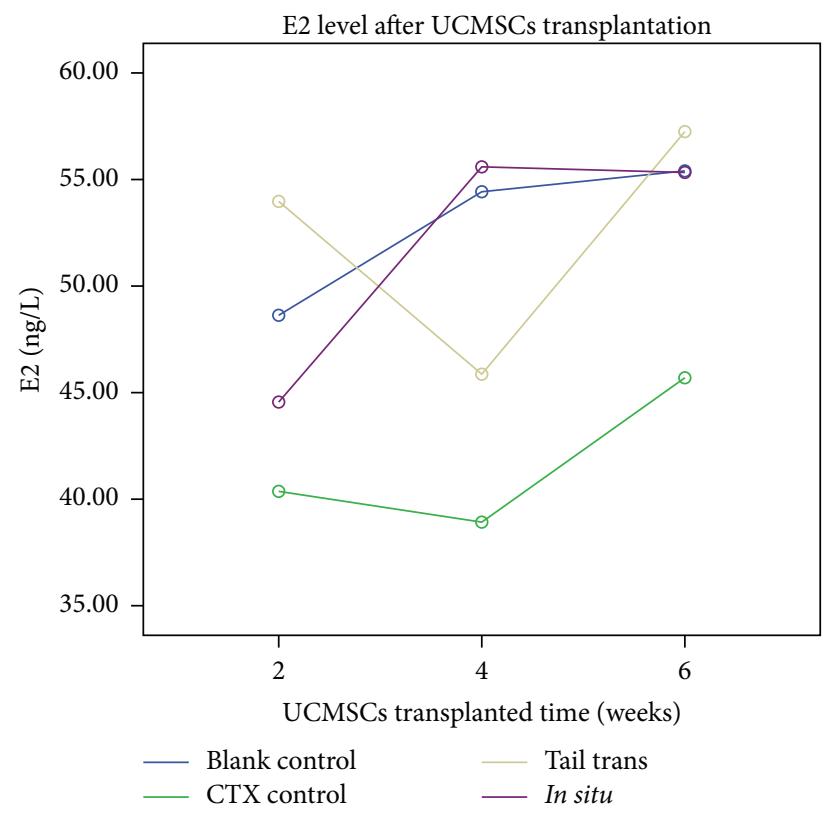

(b)

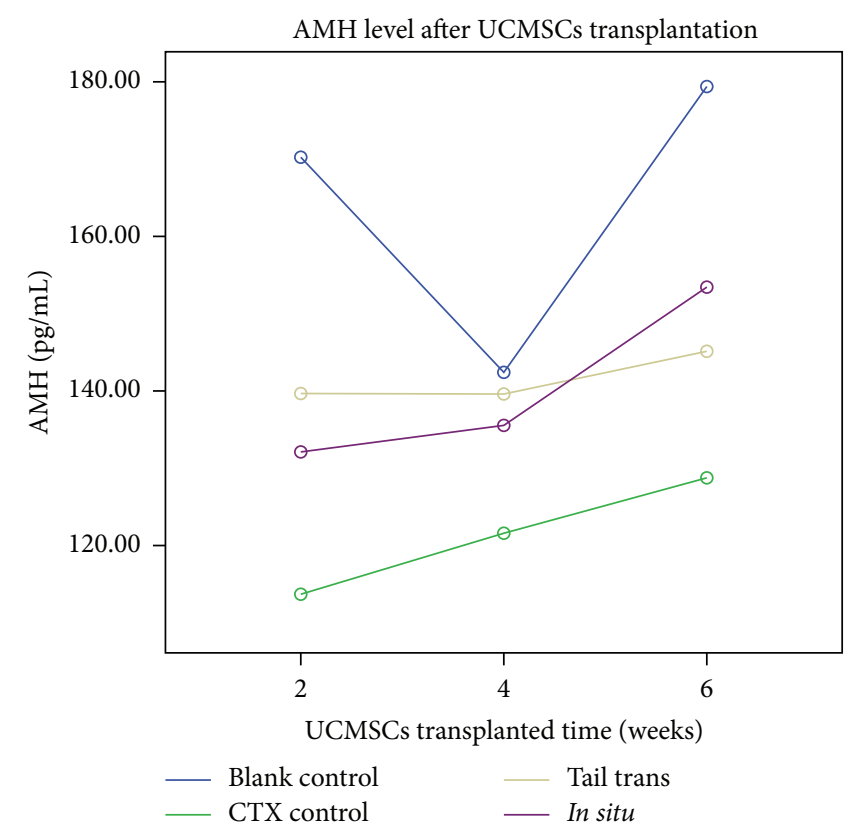

(c)

FIGURE 5: The trend for average level of FSH, AMH, and E2 in each group during six weeks after UCMSCs transplantation.

There are several advantages for UCMSCs over other MSCs, such as having poor immunogenic properties, which is attributed to their low expression of human leukocyte antigen major histocompatibility complex I (MHC I) and the absence of MHC II molecules [33, 34]. UCMSCs can be easily isolated and expanded in vitro. Additionally, they exhibit little ethical issues as compared to other types of stem cells. The cell-surface markers of UCMSCs have been characterized as positive for $\mathrm{CD} 29, \mathrm{CD} 73, \mathrm{CD} 90$, and $\mathrm{CD} 105$ and negative for CD14, CD79, CD34, and CD45, HLA-DR [15, 35]. To date, only limited studies have been conducted determining the role of UCMSCs in POF therapy. Our study systematically proved its efficiency and safety in repairing the damaged rat ovary function induced by chemotherapy.

In our study, the isolated human UCMSCs expressed stem cells markers and exhibited the characteristics of mesenchymal stem cells, which was consistent with previous reports. According to our study, there was a significant reduction in cell apoptosis after the UCMSCs transplantation and improvement in the folliculogenesis in POF rat models. In order to clarify the mechanism of human UCMSCs in repairing the damaged ovarian function in POF rats, it is 
TABLE 3: Levels of serum hormone analysis and effect of UCMSCs transplantation on the serum hormone secretion. FSH, AMH, and E2 levels were determined by ELISA at two, four, and six weeks after UCMSCs transplantation. CTX control group indicates the nontransplanted POF group. Data were means \pm SD $(n=4)$.

\begin{tabular}{lccc}
\hline & 2 weeks later & 4 weeks later & 6 weeks later \\
\hline FSH (IU/L) & & & \\
Blank control & $8.695 \pm 0.284$ & $9.802 \pm 0.396$ & $8.702 \pm 1.384$ \\
CTX control & $10.864 \pm 0.559$ & $11.513 \pm 0.643$ & $11.034 \pm 0.920$ \\
Tail trans & $9.763 \pm 0.815$ & $9.985 \pm 0.501$ & $9.351 \pm 0.774$ \\
In situ trans & $11.205 \pm 1.438$ & $8.080 \pm 1.805$ & $9.493 \pm 0.670$ \\
E2 (ng/L) & & & $55.404 \pm 4.012$ \\
Blank control & $48.629 \pm 1.417$ & $54.427 \pm 9.108$ & $45.696 \pm 2.786$ \\
CTX control & $40.367 \pm 5.342$ & $38.922 \pm 8.033$ & $57.248 \pm 6.939$ \\
Tail trans & $53.974 \pm 4.045$ & $45.865 \pm 3.701$ & $55.331 \pm 5.060$ \\
In situ trans & $44.557 \pm 2.967$ & $55.594 \pm 9.580$ & $179.391 \pm 10.748$ \\
AMH (pg/mL) & & & $128.758 \pm 9.514$ \\
Blank control & $170.242 \pm 7.855$ & $142.408 \pm 20.819$ & $145.132 \pm 5.134$ \\
CTX control & $113.699 \pm 18.022$ & $121.590 \pm 5.094$ & $153.435 \pm 16.090$ \\
Tail trans & $139.670 \pm 5.843$ & $139.603 \pm 19.995$ & $135.545 \pm 9.847$ \\
In situ trans & $132.111 \pm 12.203$ & & \\
\hline
\end{tabular}

TABLE 4: Counts of different stages follicles were presented as means $\pm \mathrm{SD}(n=3)$ (primordial, primary, and early antral follicles of POF group versus UCMSCs transplanted group: $p>0.05$, secondary follicles of POF group versus UCMSCs transplanted group: $p<$ $0.05)$.

\begin{tabular}{lcccc}
\hline & Primordial & Primary & Secondary & Early antral \\
\hline Blank control & $18.3 \pm 4.0$ & $8.7 \pm 1.2$ & $8.0 \pm 0$ & $38.7 \pm 2.3$ \\
CTX control & $17.7 \pm 9.3$ & $6.0 \pm 1.0$ & $2.0 \pm 1.0$ & $36.0 \pm 11.4$ \\
Tail trans & $25.3 \pm 18.0$ & $11.7 \pm 5.5$ & $6.7 \pm 1.2$ & $43.0 \pm 4.4$ \\
In situ trans & $20.7 \pm 7.8$ & $8.0 \pm 2.6$ & $6.0 \pm 1.7$ & $52.7 \pm 6.0$ \\
\hline$p$ & $\mathrm{~ns}$ & $\mathrm{~ns}$ & $<0.05$ & $\mathrm{~ns}$ \\
\hline
\end{tabular}

imperative to trace the fate of the transplanted cells. The UCMSCs, used in our study, were derived from the umbilical cord of new born male, which helped us to detect its location and growth situation in the ovarian tissue by labeling its $\mathrm{X}$ and Y chromosome by FISH.

According to FISH results, the transplanted UCMSCs resided in ovarian tissue and survived for at least eight weeks. The cell homing ability of the UCMSCs was based on the expressing mounts of cell adhesion molecules, which aids in the migration of these cells to the target tissues [36]. According to previous reports, UCMSCs could reduce GC apoptosis by affecting its G-protein coupled receptor protein signaling and MAPK pathways, both of which are important for follicle and oocyte growth [37]. UCMSCs could secrete a number of angiogenic growth factors, such as hepatocyte growth factor (HGF), vascular endothelial growth factor (VEGF), placental growth factor (PGF), and TGF- $\beta$. Many of these factors have been found to have the ability to repair the damaged ovarian function [38]. We speculated that the improved ovarian function in POF rat model was more likely due to the cytokines produced by UCMSCs via paracrine action. However, this speculation needs further investigation.

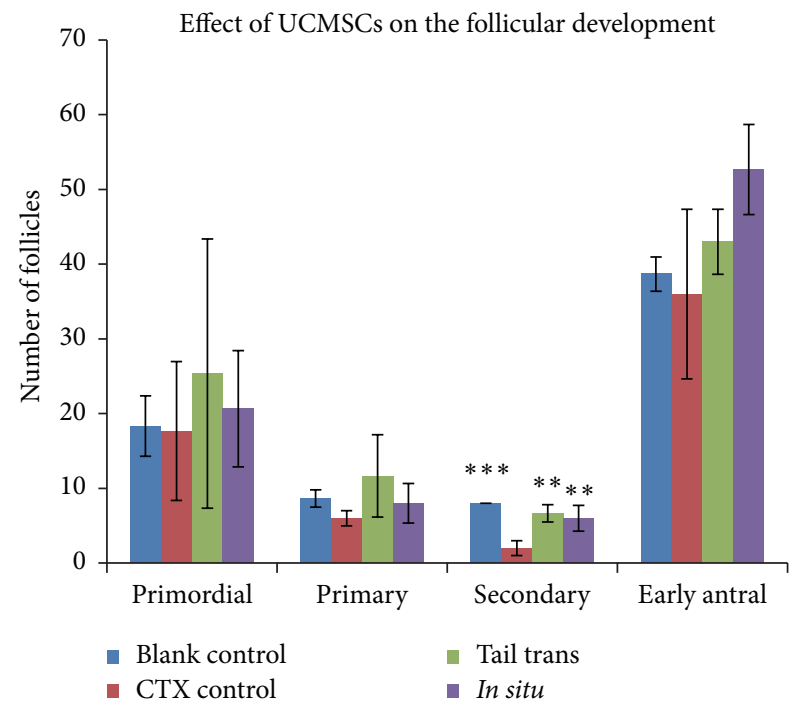

FIGURE 6: Effect of UCMSCs transplantation on follicular development. Ovarian $\mathrm{H} \& \mathrm{E}$ staining sections were from blank control group, CTX control group, and UCMSCs transplantation groups (by tail intravenously or in situ). Follicles were numbered and classified. Data were means \pm SD of counts of different stages follicles in three experiments (secondary follicles of UCMSCs treatment groups versus POF control group: $p<0.01$; primordial, primary, and early antral follicles of UCMSCs treatment groups versus POF control group: $p>0.05, n=3) .{ }^{* *} p<0.01 ;{ }^{* * *} p<0.001$.

Endocrine system produces a variety of steroidal hormones, which could regulate follicle growth and survival by paracrine or autocrine mechanism. Disordered hormone secretion is not the only cause but also the outcome of POF pathological process [39]. FSH is produced by adenohypophysis, which exerts its action on the ovary by stimulating follicles growth. FSH receptors (FSHR) are mainly expressed 


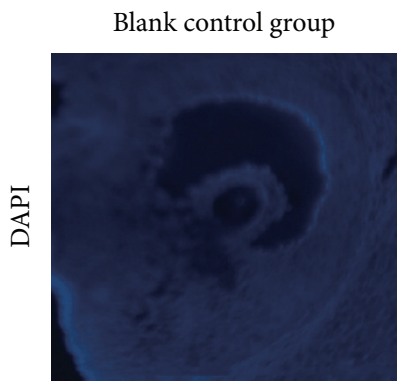

(a)

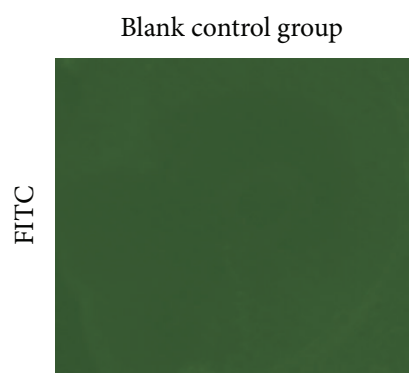

(e)

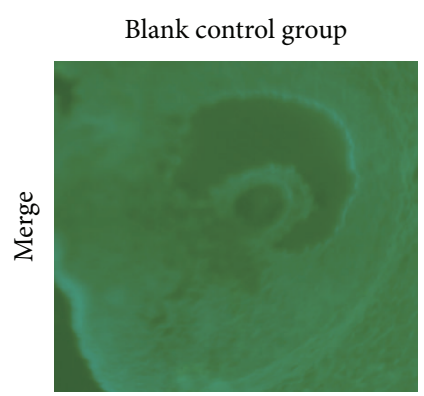

(i)

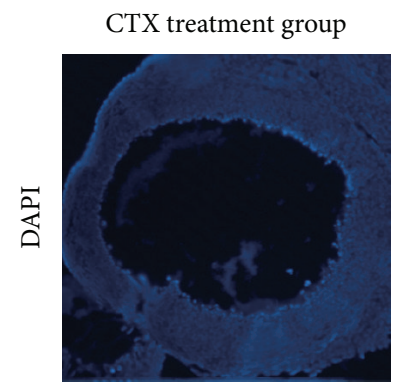

(b)

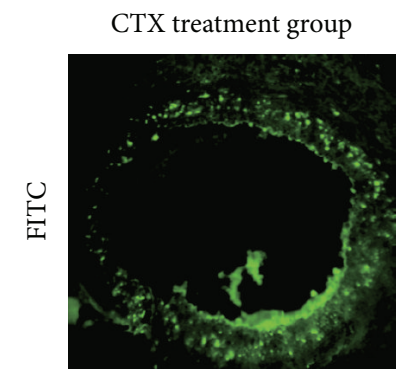

(f)

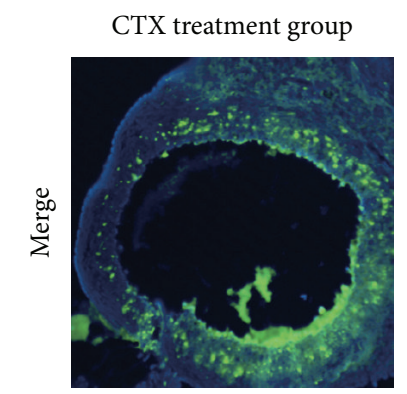

(j)

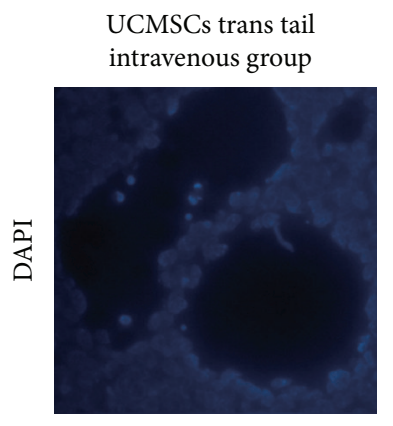

(c)

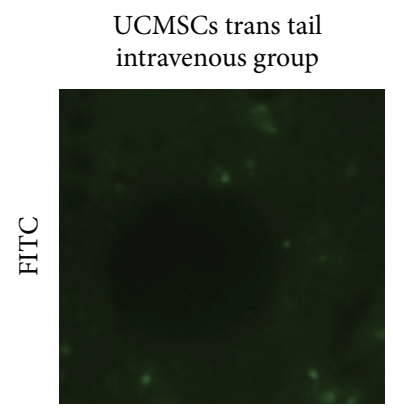

(g)

UCMSCs trans tail intravenous group

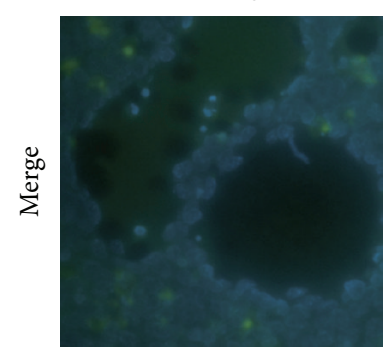

(k)

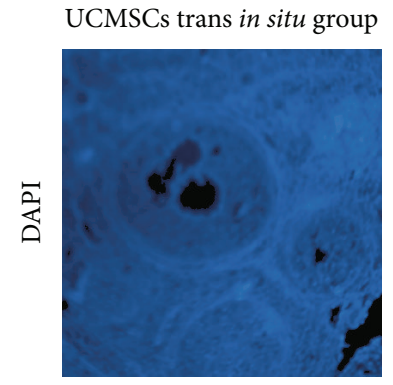

(d)

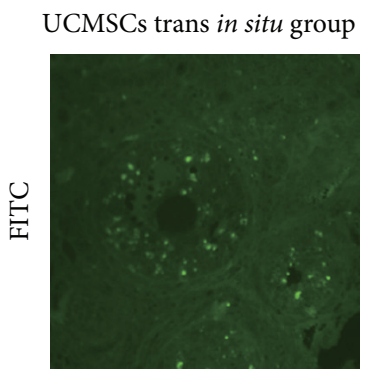

(h)

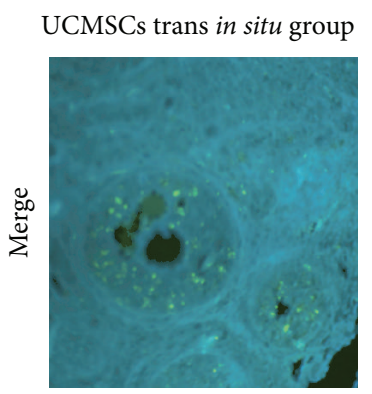

(1)

FIGURE 7: Cell apoptosis assay: UCMSCs transplantation reduced cell apoptosis of ovaries in CTX-induced POF rats. (a, b, c, d) Blue fluorescence indicates cell nucleus stained by DAPI (blank control group, CTX treatment group, tail intravenous trans group, and in situ UCMSCs trans group). (e, f, g, h) Green fluorescence (FITC) stained cells illustrating the degree and site of apoptosis of cells in the four groups. (i, j, k, l) merged the two stainings.

on GCs [40]. Increased level of FSH could accelerate follicles storage depletion; both the cause and the marker diminish ovary reserve [41, 42]. E2 exerts negative feedback on FSH production in the hypothalamus-pituitary glands axis, which is mainly produced by GCs. Overapoptosis of GCs induced by chemotherapeutic agents often results in decreased E2 level. The interrupted negative feedback results in uncontrolled increase in the level of FSH [43]. AMH, which is expressed by GCs of the primary follicles or early antral follicles, acts as an inhibitory growth factor in the ovary during the early stages of folliculogenesis [44]. Usually, the level of AMH is stable during the whole menstrual cycle. Its level strongly correlates with the size of the follicle pool [45].

Our study gave a comprehensive report on the disordered steroidal hormone secretion in POF rats induced by CTX administration. Human UCMSCs transplantation improved the disturbed endocrine secretion system to some degree, which is an important mechanism for improving the damaged ovary function. We illustrated the hormone secretion in hypothalamus-adenohypophysis-ovarian axis during the pathological process of POF and the possible therapeutic mechanism of UCMSCs (Figure 9).

\section{Strengths and Limitations}

There are several strengths and limitations of our present study. We monitored the effects of CTX administration on endocrine system function at several CTX posttreatment intervals and concluded that ovarian function declined with time after treatment. Analysis of the change in hormone levels after human UCMSCs transplantation indicated that restoration of the ovarian function indeed takes place to some 


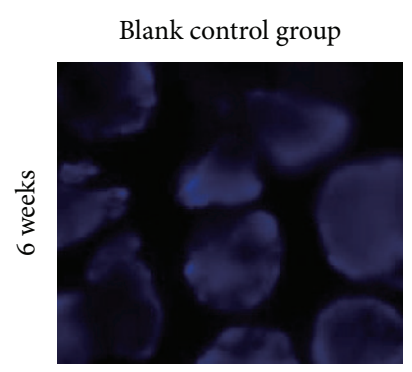

(a)

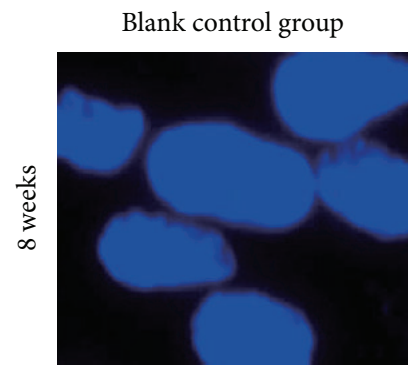

(e)

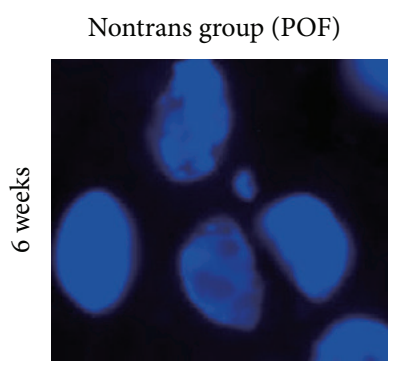

(b)

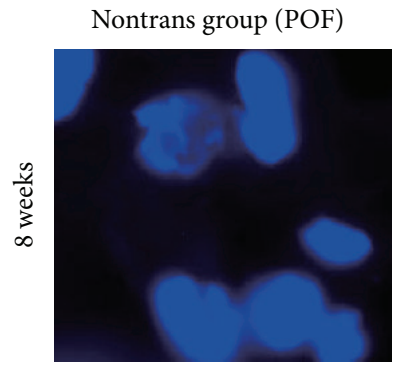

(f)

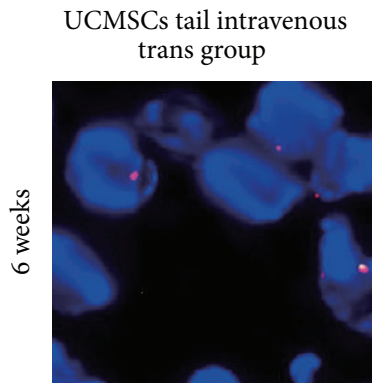

(c)

UCMSCs tail intravenous

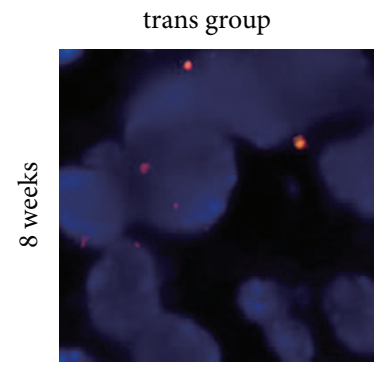

(g)
UCMSCs in situ trans group

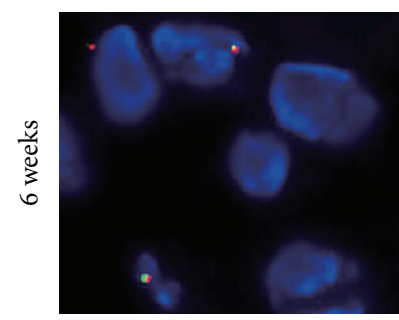

(d)

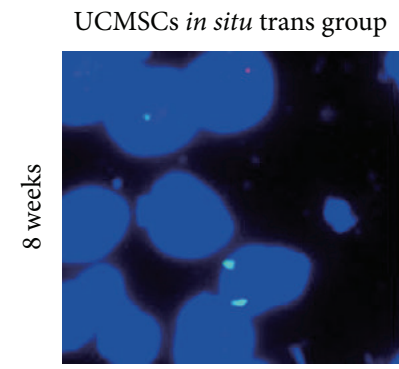

(h)

FIGURE 8: FISH analysis of UCMSCs, tracking of the transplanted human UCMSCs in rats ovarian tissue. (a, b, c, d) Distribution of human UCMSCs in rats ovaries after transplantation for six weeks (blank control group, CTX control group, tail intravenous trans group, and in situ trans group). (e, $\mathrm{f}, \mathrm{g}, \mathrm{h}$ ) Distribution of human UCMSCs in rats ovaries after transplantation for eight weeks. The location of the UCMSCs was determined using orange (SRY/Y chromosome) and green (CEP/X chromosome) signals. Double-labeled staining (red and green fluorescence) cells were defined as human UCMSCs.

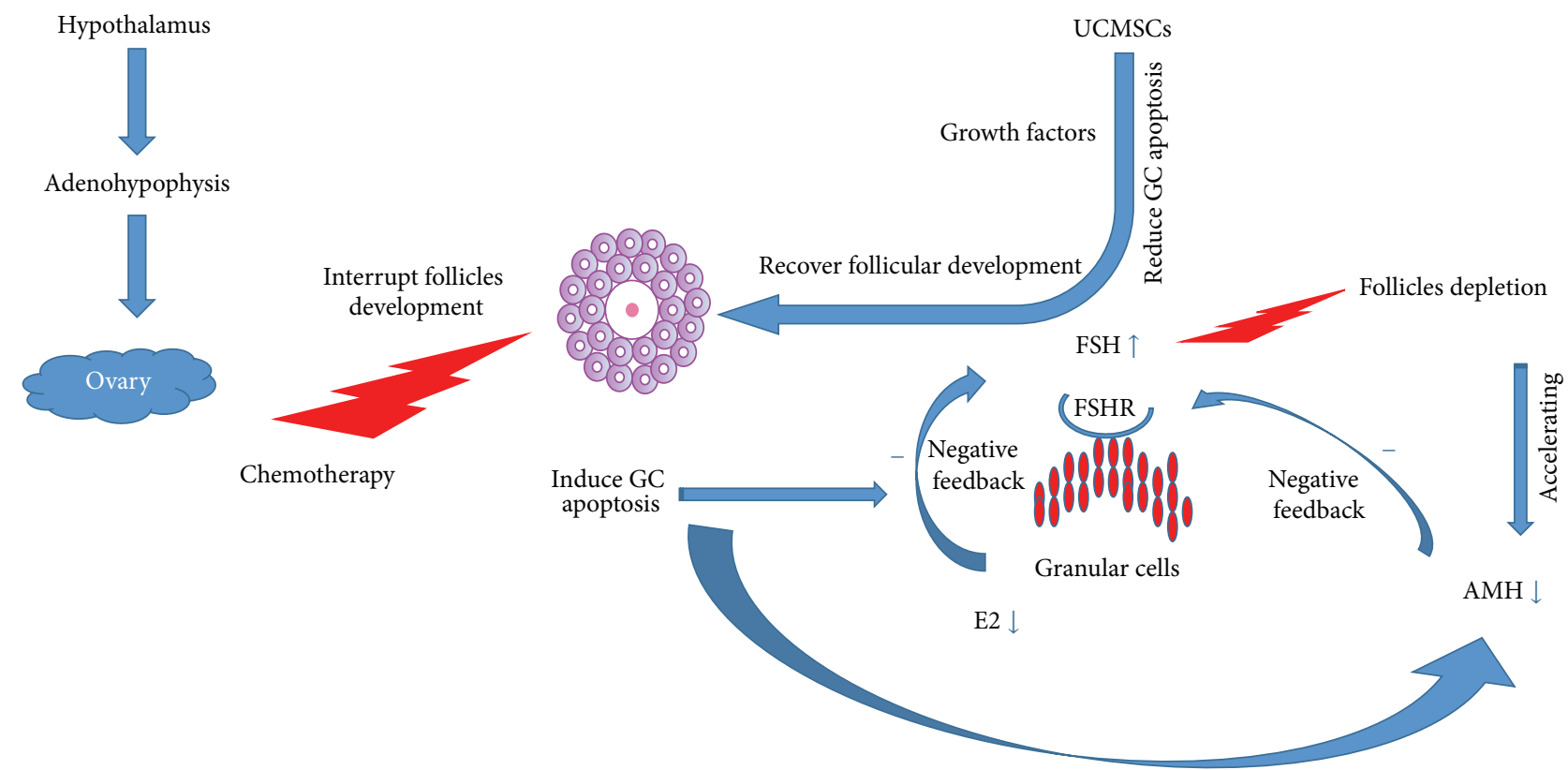

FIGURE 9: The network of steroidal hormones regulation system during pathological process of POF and UCMSCs therapeutic mechanism. With the hypothalamus regulation, adenohypophysis could synthesize and produce gonadotropins (FSH), which could stimulate follicles growth. GCs of the growth follicles could produce E2, having a feedback negative effect on FSH production, thus reducing follicles excessive growth and avoiding their depletion. AMH could also inhibit FSH production. The negative feedback effect of E2 and AMH on the follicles growth was interrupted by CTX administration. Lack of inhibition of E2 and AMH causes uncontrolled elevating FSH level, which promotes follicles pool exhausted very soon, leading to POF. The transplanted human UCMSCs may secrete kinds of growth factors, which could rescue the overapoptosis of GCs and ultimately improve follicles development and retargeted hormonal balance. 
degree. An ideal therapeutic window for UCMSCs transplantation should be suggested by further study. Additionally, UCMSCs used in our experiment were derived from male baby; Y chromosome was considered as its marker, which could avoid any effects of the cell label on stem cells' survival.

The tumorigenic potential of stem cells therapy has always been an obstacle in its broad application. We traced the transplanted human UCMSCs in rat ovarian tissues at different time points. Although our results revealed that the transplanted human UCMSCs could survive for a long time without obvious proliferation, our experiment did not last long enough to follow the ultimate fate of these cells. Additionally, we could not exclude the possible tumorigenicity potential of these cells. We did not investigate the fertility of the rat model following our current CTX administration protocol and UCMSCs therapy. Further and deeper investigation is required to better understand the exact mechanism of the therapeutic potential of human UCMSCs in POF.

\section{Conclusion}

In conclusion, our present study systemically studied the efficiency of human UCMSCs in POF rat models, such as human UCMSCs transplantation improving the disturbed endocrine secretion system, reducing the cell apoptosis of ovary, and improving the folliculogenesis. The safety and involved mechanisms of human UCMSCs in POF treatment still need further investigation.

\section{Competing Interests}

The authors have declared that no competing interests existed.

\section{Acknowledgments}

This study was supported by the Science and Technology Supporting Social Development Fund of Jiangsu Province, China (BE2012653).

\section{References}

[1] C. B. Coulam, "Premature gonadal failure," Fertility and Sterility, vol. 38, no. 6, pp. 645-655, 1982.

[2] C. J. Davis, R. M. Davison, N. N. Payne, C. H. Rodeck, and G. S. Conway, "Female sex preponderance for idiopathic familial premature ovarian failure suggests an $\mathrm{X}$ chromosome defect: opinion," Human Reproduction, vol. 15, no. 11, pp. 2418-2422, 2000.

[3] R. Goswami, D. Goswami, M. Kabra, N. Gupta, S. Dubey, and V. Dadhwal, "Prevalence of the triple X syndrome in phenotypically normal women with premature ovarian failure and its association with autoimmune thyroid disorders," Fertility and Sterility, vol. 80, no. 4, pp. 1052-1054, 2003.

[4] J. L. Simpson and A. Rajkovic, "Ovarian differentiation and gonadal failure," American Journal of Medical Genetics, vol. 89, no. 4, pp. 186-200, 1999.

[5] M. F. H. Brougham and W. H. B. Wallace, "Subfertility in children and young people treated for solid and haematological malignancies," British Journal of Haematology, vol. 131, no. 2, pp. 143-155, 2005.

[6] J. E. Fortune, R. A. Cushman, C. M. Wahl, and S. Kito, "The primordial to primary follicle transition," Molecular and Cellular Endocrinology, vol. 163, no. 1-2, pp. 53-60, 2000.

[7] C. H. de Koning, J. McDonnell, A. P. N. Themmen, F. H. de Jong, R. Homburg, and C. B. Lambalk, "The endocrine and follicular growth dynamics throughout the menstrual cycle in women with consistently or variably elevated early follicular phase FSH compared with controls," Human Reproduction, vol. 23, no. 6, pp. 1416-1423, 2008.

[8] J. Kwee, R. Schats, J. McDonnell, A. Themmen, F. de Jong, and C. Lambalk, "Evaluation of anti-Müllerian hormone as a test for the prediction of ovarian reserve," Fertility and Sterility, vol. 90, no. 3, pp. 737-743, 2008.

[9] Y. E. Sukur, I. Balik Kivancli, and B. Ozmen, "Ovarian aging and premature ovarian failure," Journal of the Turkish German Gynecological Association, vol. 15, no. 3, pp. 190-196, 2014.

[10] X. Fu, Y. He, C. Xie, and W. Liu, "Bone marrow mesenchymal stem cell transplantation improves ovarian function and structure in rats with chemotherapy-induced ovarian damage," Cytotherapy, vol. 10, no. 4, pp. 353-363, 2008.

[11] S. Kestendjieva, D. Kyurkchiev, G. Tsvetkova et al., "Characterization of mesenchymal stem cells isolated from the human umbilical cord," Cell Biology International, vol. 32, no. 7, pp. 724732, 2008.

[12] S. Karahuseyinoglu, O. Cinar, E. Kilic et al., "Biology of stem cells in human umbilical cord stroma: in situ and in vitro surveys," STEM CELLS, vol. 25, no. 2, pp. 319-331, 2007.

[13] U. Nekanti, L. Mohanty, P. Venugopal, S. Balasubramanian, S. Totey, and M. Ta, "Optimization and scale-up of Wharton's jellyderived mesenchymal stem cells for clinical applications," Stem Cell Research, vol. 5, no. 3, pp. 244-254, 2010.

[14] C. Qiao, W. Xu, W. Zhu et al., "Human mesenchymal stem cells isolated from the umbilical cord," Cell Biology International, vol. 32, no. 1, pp. 8-15, 2008.

[15] M. Yan, M. Sun, Y. Zhou et al., "Conversion of human umbilical cord mesenchymal stem cells in Wharton's jelly to dopamine neurons mediated by the $\operatorname{Lmx}$ la and neurturin in vitro: potential therapeutic application for Parkinson's disease in a rhesus monkey model," PLoS ONE, vol. 8, no. 5, Article ID e64000, 2013.

[16] H. Lin, R. Xu, Z. Zhang, L. Chen, M. Shi, and F.-S. Wang, "Implications of the immunoregulatory functions of mesenchymal stem cells in the treatment of human liver diseases," Cellular and Molecular Immunology, vol. 8, no. 1, pp. 19-22, 2011.

[17] N. Souidi, M. Stolk, and M. Seifert, "Ischemia-reperfusion injury: beneficial effects of mesenchymal stromal cells," Current Opinion in Organ Transplantation, vol. 18, no. 1, pp. 34-43, 2013.

[18] Y. Yan, W. Xu, H. Qian et al., "Mesenchymal stem cells from human umbilical cords ameliorate mouse hepatic injury in vivo," Liver International, vol. 29, no. 3, pp. 356-365, 2009.

[19] G.-Y. Xiao, I.-H. Liu, C.-C. Cheng et al., "Amniotic fluid stem cells prevent follicle atresia and rescue fertility of mice with premature ovarian failure induced by chemotherapy," PLoS ONE, vol. 9, no. 9, Article ID e106538, 2014.

[20] K. Zou, Z. Yuan, Z. Yang et al., "Production of offspring from a germline stem cell line derived from neonatal ovaries," Nature Cell Biology, vol. 11, no. 5, pp. 631-636, 2009.

[21] M. Myers, K. L. Britt, N. G. M. Wreford, F. J. P. Ebling, and J. B. Kerr, "Methods for quantifying follicular numbers within the mouse ovary," Reproduction, vol. 127, no. 5, pp. 569-580, 2004. 
[22] W. Chemaitilly, A. C. Mertens, P. Mitby et al., "Acute ovarian failure in the childhood cancer survivor study," Journal of Clinical Endocrinology and Metabolism, vol. 91, no. 5, pp. 17231728, 2006.

[23] C. A. Sklar, A. C. Mertens, P. Mitby et al., "Premature menopause in survivors of childhood cancer: a report from the childhood cancer survivor study," Journal of the National Cancer Institute, vol. 98, no. 13, pp. 890-896, 2006.

[24] R. Iorio, A. Castellucci, G. Ventriglia et al., "Ovarian toxicity: from environmental exposure to chemotherapy," Current Pharmaceutical Design, vol. 20, no. 34, pp. 5388-5397, 2014.

[25] E. Chavakis, C. Urbich, and S. Dimmeler, "Homing and engraftment of progenitor cells: a prerequisite for cell therapy," Journal of Molecular and Cellular Cardiology, vol. 45, no. 4, pp. 514-522, 2008.

[26] E. Markström, E. C. Svensson, R. Shao, B. Svanberg, and H. Billig, "Survival factors regulating ovarian apoptosis-dependence on follicle differentiation," Reproduction, vol. 123, no. 1, pp. 2330, 2002.

[27] M. E. B. Clowse, M. A. Behera, C. K. Anders et al., "Ovarian preservation by $\mathrm{GnRH}$ agonists during chemotherapy: a metaanalysis," Journal of Women's Health, vol. 18, no. 3, pp. 311-319, 2009.

[28] S. K. Nabhan, M. A. Bitencourt, M. Duval et al., "Fertility recovery and pregnancy after allogeneic hematopoietic stem cell transplantation in Fanconi anemia patients," Haematologica, vol. 95, no. 10, pp. 1783-1787, 2010.

[29] M. Sun, S. Wang, Y. Li et al., "Adipose-derived stem cells improved mouse ovary function after chemotherapy-induced ovary failure," Stem Cell Research and Therapy, vol. 4, no. 4, article 80, 2013.

[30] T. Liu, Y. Huang, L. Guo, W. Cheng, and G. Zou, "CD44+/CD105+ human amniotic fluid mesenchymal stem cells survive and proliferate in the ovary long-term in a mouse model of chemotherapy-induced premature ovarian failure," International Journal of Medical Sciences, vol. 9, no. 7, pp. 592-602, 2012.

[31] D. Lai, F. Wang, Z. Dong, and Q. Zhang, "Skin-derived mesenchymal stem cells help restore function to ovaries in a premature ovarian failure mouse model," PLoS ONE, vol. 9, no. 5, Article ID e98749, 2014.

[32] T. Liu, Y. Huang, J. Zhang et al., “Transplantation of human menstrual blood stem cells to treat premature ovarian failure in mouse model," Stem Cells and Development, vol. 23, no. 13, pp. 1548-1557, 2014.

[33] K. Bieback and I. Brinkmann, "Mesenchymal stromal cells from human perinatal tissues: from biology to cell therapy," World Journal of Stem Cells, vol. 2, no. 4, pp. 81-92, 2010.

[34] Y. Liu, R. Mu, S. Wang et al., "Therapeutic potential of human umbilical cord mesenchymal stem cells in the treatment of rheumatoid arthritis," Arthritis Research and Therapy, vol. 12, no. 6, article R210, 2010.

[35] M. Dominici, K. Le Blanc, I. Mueller et al., "Minimal criteria for defining multipotent mesenchymal stromal cells. The International Society for Cellular Therapy position statement," Cytotherapy, vol. 8, no. 4, pp. 315-317, 2006.

[36] C. Shen, P. Lie, T. Miao et al., "Conditioned medium from umbilical cord mesenchymal stem cells induces migration and angiogenesis," Molecular Medicine Reports, vol. 12, no. 1, pp. 2030, 2015.
[37] S. Wang, L. Yu, M. Sun et al., "The therapeutic potential of umbilical cord mesenchymal stem cells in mice premature ovarian failure," BioMed Research International, vol. 2013, Article ID 690491, 12 pages, 2013.

[38] S. H. Abd-Allah, S. M. Shalaby, H. F. Pasha et al., "Mechanistic action of mesenchymal stem cell injection in the treatment of chemically induced ovarian failure in rabbits," Cytotherapy, vol. 15, no. 1, pp. 64-75, 2013.

[39] M. de Vos, P. Devroey, and B. C. Fauser, "Primary ovarian insufficiency," The Lancet, vol. 376, no. 9744, pp. 911-921, 2010.

[40] L. A. Lazaros, E. G. Hatzi, C. E. Pamporaki et al., "The ovarian response to standard gonadotrophin stimulation depends on FSHR, SHBG and CYP19 gene synergism," Journal of Assisted Reproduction and Genetics, vol. 29, no. 11, pp. 1185-1191, 2012.

[41] F. J. Broekmans, E. A. H. Knauff, E. R. te Velde, N. S. Macklon, and B. C. Fauser, "Female reproductive ageing: current knowledge and future trends," Trends in Endocrinology and Metabolism, vol. 18, no. 2, pp. 58-65, 2007.

[42] F. J. Broekmans, M. R. Soules, and B. C. Fauser, "Ovarian aging: mechanisms and clinical consequences," Endocrine Reviews, vol. 30, no. 5, pp. 465-493, 2009.

[43] K. Yacobi, A. Wojtowicz, A. Tsafriri, and A. Gross, "Gonadotropins enhance caspase-3 and -7 activity and apoptosis in the theca-interstitial cells of rat preovulatory follicles in culture," Endocrinology, vol. 145, no. 4, pp. 1943-1951, 2004.

[44] C. Weenen, J. S. E. Laven, A. R. M. von Bergh et al., "AntiMüllerian hormone expression pattern in the human ovary: potential implications for initial and cyclic follicle recruitment," Molecular Human Reproduction, vol. 10, no. 2, pp. 77-83, 2004.

[45] J. A. Visser, I. Schipper, J. S. E. Laven, and A. P. N. Themmen, "Anti-Müllerian hormone: an ovarian reserve marker in primary ovarian insufficiency," Nature Reviews Endocrinology, vol. 8, no. 6, pp. 331-341, 2012. 


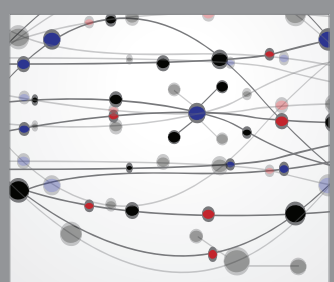

The Scientific World Journal
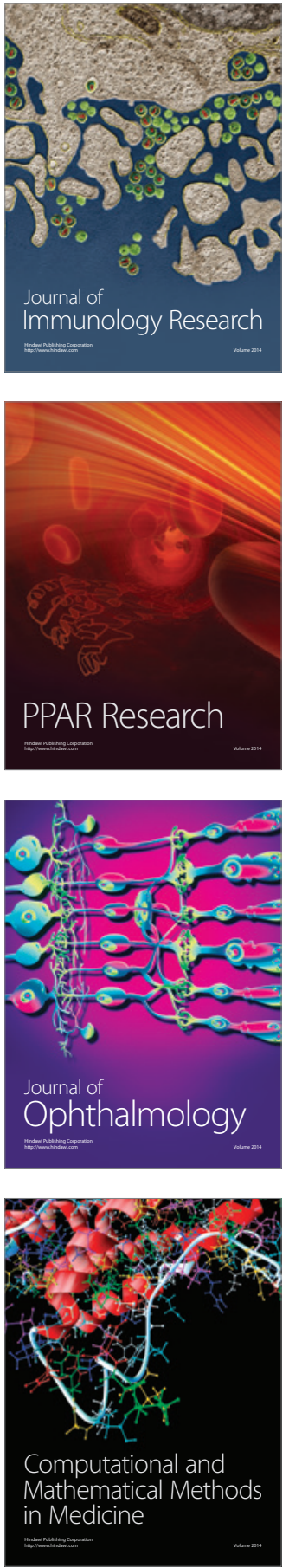

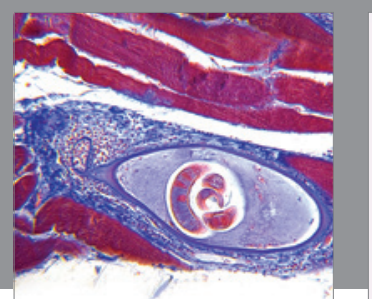

Gastroenterology Research and Practice

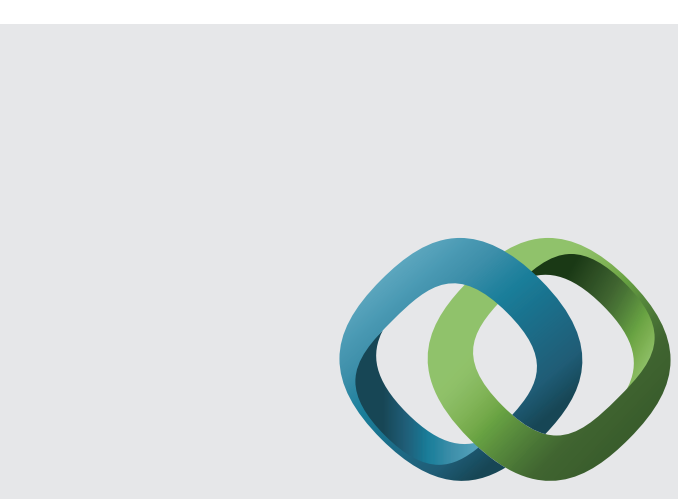

\section{Hindawi}

Submit your manuscripts at

http://www.hindawi.com
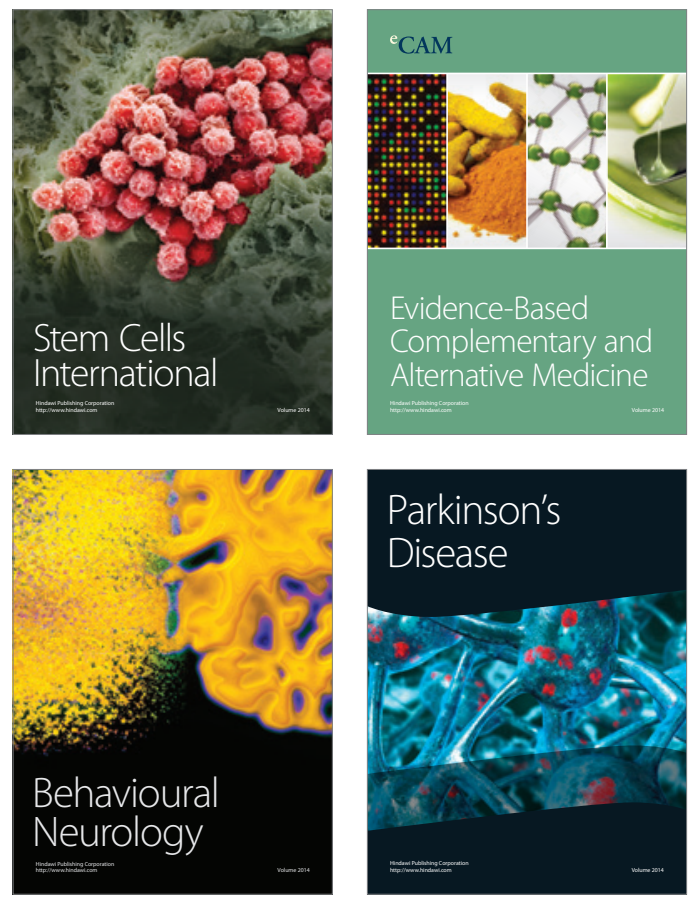
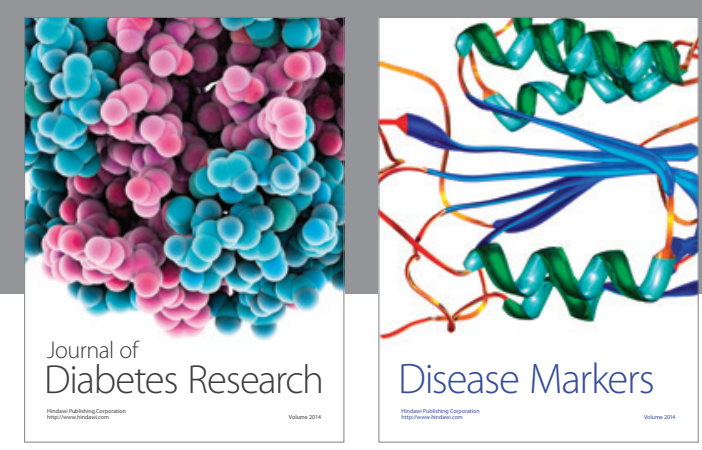

Disease Markers
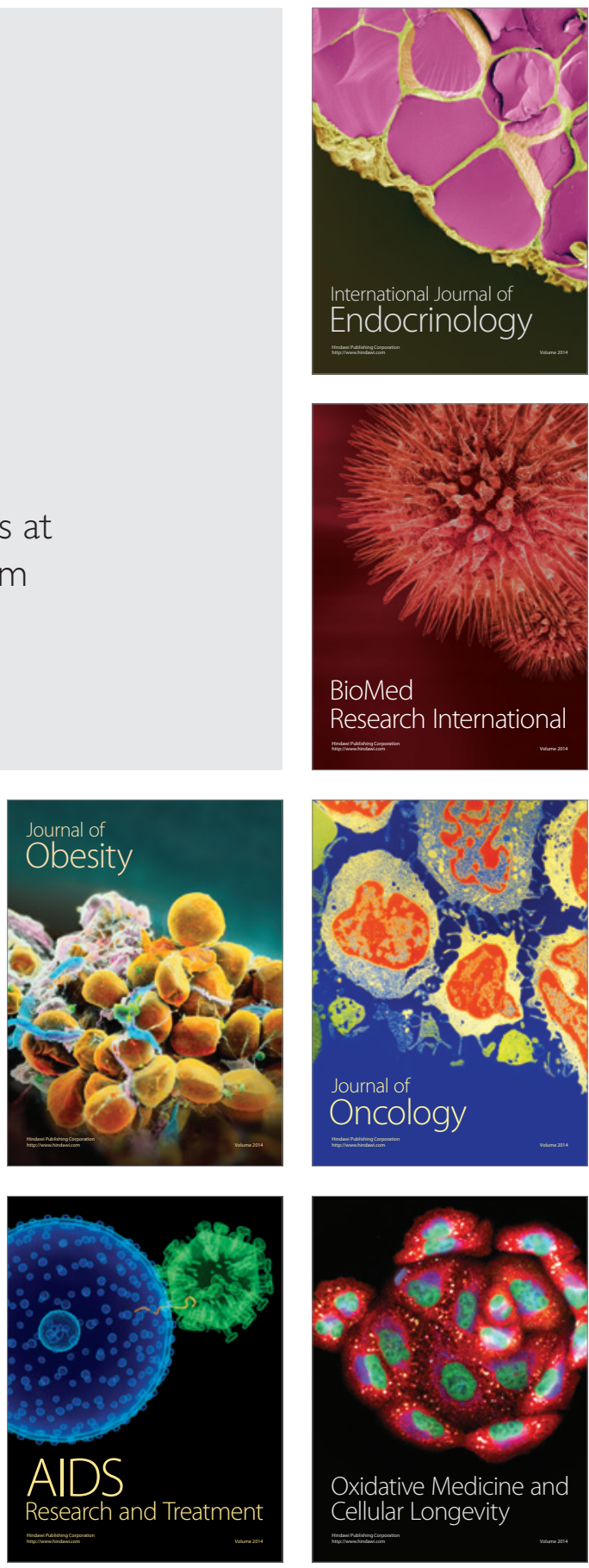